\title{
The Role of CD18-mediated Adhesion in Neutrophil Sequestration Induced by Infusion of Activated Plasma in Rabbits
}

\author{
Claire M. Doerschuk
}

Pulmonary Research Laboratory, University of British Columbia, St. Paul's Hospital, Vancouver, British Columbia, Canada

\begin{abstract}
Infusion of activated plasma induces neutropenia and sequestration of neutrophils within the microvasculature. This study examined the role of the adhesion glycoprotein complex, CD11/CD18, in this sequestration. Rabbits pretreated with either the anti-CD18 monoclonal antibody (mAb) 60.3 or saline were given infusions of zymosan-activated plasma (ZAP) or saline. The effect of mAb 60.3 on the changes in circulating neutrophil counts, radiolabeled neutrophil kinetics in the lung, and the pulmonary microvascular accumulation of neutrophils induced by ZAP infusion was determined. The data show that pretreatment with $\mathrm{mAb}$ 60.3 did not inhibit either the rate of onset or the severity of the neutropenia but prevented the sustained neutropenia. In addition, mAb 60.3 completely prevented the ZAP-induced changes in radiolabeled neutrophil kinetics and largely inhibited the accumulation of neutrophils within the capillaries and the small vessels when evaluated after $15 \mathrm{~min}$ of ZAP infusion. We conclude that neutrophil accumulation is a two-step process, the first occurring through a CD18-independent mechanism that may involve a stimulus-induced decrease in neutrophil deformability and acts to slow neutrophil transit through the lung. The second step requires CD18-dependent adhesion and is needed for prolonged accumulation of neutrophils within the pulmonary microvasculature.
\end{abstract}

The lung contains a large marginated pool of neutrophils within the capillary bed. Because neutrophils are normally more than 1,000 times less deformable than erythrocytes (RBC), neutrophils require more time than $\mathrm{RBC}$ to pass through capillaries that are narrower than the neutrophil's spherical diameter (1-8). Previous work from our laboratory and others has shown that the multisegmented nature of the pulmonary capillary bed allows the RBC to bypass segments that are filled by the temporarily obstructing neutrophils, concentrating the neutrophils with respect to the $\mathrm{RBC}$ to form the marginated pool $(2,5-9)$. Several groups of investigators have suggested that a further reduction in deformability may explain the pulmonary sequestration of neutrophils produced by an infusion of activated plasma $(9,10)$. However, this sequestration could also be explained by an increase in adhesion between neutrophils and endothelial cells.

The leukocyte adhesion complex, CD11/CD18, mediates neutrophil-endothelial cell adherence in vitro $(11,12)$. In vivo, this complex is important in neutrophil emigration to-

(Received in original form November 11,1990 and in revised form January 8, 1992)

Address correspondence to: Claire M. Doerschuk, M.D., Riley Hospital for Children, Room 2600, Indiana University, 702 Barnhill Dr., Indianapolis, IN 46202-5225.

Abbreviations: ${ }^{51}$ chromium-labeled neutrophils, Cr-PMN; monoclonal antibody, mAb; erythrocyte(s), RBC; ${ }^{99 m}$ technetium-labeled RBC, Tc-RBC; zymosan-activated plasma, ZAP.

Am. J. Respir, Cell Mol. Biol. Vol. 7. pp. 140-148, 1992 ward an extravascular chemotactic stimulus (13-17). However, the role of $\mathrm{CD} 11 / \mathrm{CD} 18$ in the rapid sequestration of neutrophils and in their subsequent accumulation within the microvasculature after intravascular infusion of stimuli has not been examined. The anti-CD18 monoclonal antibody (mAb) 60.3 inhibits CD18-mediated adhesion both in vitro and in vivo (11-17). The purpose of this study was to determine if pretreatment with this antibody had any effect on the neutropenia or the accumulation of neutrophils within the pulmonary microvasculature that was induced by infusion of activated plasma. The data show that mAb 60.3 pretreatment had no effect on the initial fall in circulating neutrophil counts; however, it did prevent the subsequent accumulation of neutrophils within the lungs. We suggest that the margination of neutrophils induced by the infusion of activated plasma has an initial phase that may depend on a change in cell deformability and a second phase that requires increased adhesion between neutrophils and endothelial cells.

\section{Materials and Methods}

The murine mAb 60.3 against CD18 (17) was kindly provided by Dr. John M. Harlan, University of Washington.

\section{Radiolabeling of Neutrophils and RBC}

Rabbit neutrophils were isolated using previously described methods $(6,9)$. In brief, rabbit peripheral blood was drawn into acid-citrate-dextrose. The RBC were sedimented using dextran (100,000 to $200,000 \mathrm{D})$, and residual RBC were removed by hypotonic lysis. The neutrophils were separated 
from the mononuclear cells by centrifugation through Histopaque (Sigma Chemical Co., St. Louis, MO). The neutrophils $>95 \%$ pure) were labeled with ${ }^{51}$ chromium $(\mathrm{Cr}-$ PMN) by incubation with sodium ${ }^{51}$ chromate (Merck Frosst, Montreal, Quebec) at $37^{\circ} \mathrm{C}$ for $30 \mathrm{~min}$.

Rabbit $\mathrm{RBC}$ were drawn using acid-citrate-dextrose as the anticoagulant and were labeled with ${ }^{99 m}$ technetium (TCRBC) using a Glucoscan kit (New England Nuclear, Boston, MA) (18).

\section{Preparation of Zymosan-activated Plasma (ZAP)}

ZAP was prepared by incubating heparinized rabbit plasma combined with zymosan A yeast (Z-4250; Sigma) $(5 \mathrm{mg} / \mathrm{ml}$ plasma) at $37^{\circ} \mathrm{C}$ for $30 \mathrm{~min}$. The plasma was centrifuged twice at $500 \times g$ for $10 \mathrm{~min}$ and used within $60 \mathrm{~min}$.

\section{Experimental Protocol}

New Zealand white rabbits weighing $1.4 \pm 0.2 \mathrm{~kg}$ were anesthetized using intramuscular injection of ketamine hydrochloride (60 to $80 \mathrm{mg} / \mathrm{kg}$ ) and acepromazine maleate (4 to $6 \mathrm{mg} / \mathrm{kg}$ ) with additional ketamine as required. After insertion of a catheter in the marginal ear vein, heparin (100 $\mathrm{U} / \mathrm{kg}$ ) was injected, and the animals were pretreated with $\mathrm{mAb} 60.3(2 \mathrm{mg} / \mathrm{kg}$ intravenously; $n=7)$ or saline $(n=14)$ for a total of $30 \mathrm{~min}$. After $15 \mathrm{~min}$, a catheter was placed in the aorta through the carotid artery. Three groups of animals were studied ( $n=7$ in each group):

Group 1: animals pretreated with saline that received infusions of saline.

Group 2: animals pretreated with saline that received infusions of ZAP.

Group 3: animals pretreated with mAb 60.3 that received infusions of ZAP.

After a baseline (time 0) sample of blood was drawn to determine the circulating blood cell count, intravenous infusions of ZAP (groups 2 and 3) or saline (group 1) were begun through the ear vein at a rate of $0.4 \mathrm{ml} / \mathrm{min}$. Blood samples for cell counts were taken after 1, 2, and $3.7 \mathrm{~min}$. At $4 \mathrm{~min}$, the cardiac output was determined by the indicator dilution technique using Tc-RBC as the indicator. A bolus of Tc-RBC $(0.1 \mathrm{ml})$ was injected into the intravenous catheter as arterial blood was sampled at 0.5 -s intervals into preweighed tubes using a fraction collector. At $5 \mathrm{~min}$, the Cr-PMN (5 to 16 $\times 10^{6} \mathrm{Cr}-\mathrm{PMN} / \mathrm{animal}$ ) that were also pretreated with $\mathrm{mAb} 60.3(100 \mu \mathrm{g} / \mathrm{ml}$, group 3) or saline for $15 \mathrm{~min}$ (groups 1 and 2) were injected intravenously. Additional Tc-RBC were given arterially to mark the blood volume. ${ }^{125} \mathrm{I}$-labeled macroaggregated albumin was injected intravenously to mark regional pulmonary blood flow. At $7 \mathrm{~min}$, blood was sampled for circulating cell counts and for the fraction of $\mathrm{Cr}$ PMN that was circulating (2-min recovery of Cr-PMN). At $15 \mathrm{~min}$, blood samples for cell counts and reference levels of circulating radioisotope counts were taken. Saturated potassium chloride was injected arterially to stop the animal's heart, the chest was opened, and the base of the heart was rapidly tied off to prevent blood loss from the lungs. The trachea was opened, and $6 \%$ glutaraldehyde in phosphate buffer was instilled. After 20 to $30 \mathrm{~min}$, the thoracic organs were removed. The lungs were cut into five slices of equal height in the plane perpendicular to the gravitational field. The slices were cut into a total of 24 pieces (slices 1 through 3 into three pieces each, slice 4 into two pieces, and slice 5 into one piece for each lung) and placed in preweighed scintillation vials. The liver was removed after clamping the major vessels to prevent loss of blood, and the liver, spleen, kidney, bowel, and reference blood and plasma samples, as well as the lung pieces, were counted in a Beckman gamma counter linked to an IBM computer for separation of multiple isotopes and correction for radiodecay.

\section{Calculations}

The calculations are summarized in Table 1 . The blood volume in each lung piece was calculated by dividing the $\mathrm{Tc}$ $\mathrm{RBC} \mathrm{cpm}$ by the number of $\mathrm{Tc}-\mathrm{RBC} \mathrm{cpm} / \mathrm{ml}$ reference blood. The total pulmonary blood volume was the sum of the blood volume in each piece. The cardiac output was determined from the time versus concentration curves, using the indicator dilution technique (19). The regional pulmonary blood flow was calculated by multiplying the cardiac output by the fraction of the total ${ }^{125}$ I-labeled macroaggregated albumin that lodged in each piece. The RBC transit time was calculated by dividing the blood volume by the blood flow for the whole lung and for each lung piece $(6,7,9)$.

The Cr-PMN distribution in the lungs and other organs was determined by dividing the sum of the noncirculating (retained) Cr-PMN cpm (the total Cr-PMN cpm minus those present due to circulating blood) in each organ by the total Cr-PMN cpm injected. The Cr-PMN recovery in the blood after either 2 or $10 \mathrm{~min}$ of circulation was obtained by multiplying the $\mathrm{Cr}-\mathrm{PMN} \mathrm{cpm} / \mathrm{g}$ blood by the rabbit's total blood volume $(60 \mathrm{ml} / \mathrm{kg}$ body weight) and subtracting the ${ }^{51} \mathrm{Cr} \mathrm{cpm}$ that were free in the plasma. The ratio of the size of the marginated to circulating Cr-PMN pool was calculated by dividing the Cr-PMN recovery in the lungs by that in the blood.

The regional Cr-PMN percent retention within the lung was calculated by dividing the number of Cr-PMN retained in each lung piece after $10 \mathrm{~min}$ of circulation by the number that were delivered to the piece $(6,9)$. The number that were retained was equal to the noncirculating $\mathrm{Cr}-\mathrm{PMN} \mathrm{cpm}$ in the lung piece. The number that were delivered was equal to the total number of Cr-PMN injected times the blood flow to that piece.

\section{Histologic Quantitation of Neutrophil Numbers in the Lung}

To compare the size of the native unisolated neutrophil pool with the retention of radiolabeled neutrophils that had circulated for $10 \mathrm{~min}$, the number of native neutrophils was quantitated morphometrically in each rabbit. After the radioisotope levels were determined, six tissue samples were taken from each rabbit, two randomly selected from the most gravity-dependent third of the lung, two from the midregion, and two from the upper, least gravity-dependent region. The blocks were dehydrated in graded alcohols, embedded in glycol methacrylate, sectioned at $2 \mu \mathrm{m}$, and stained with toluidine blue $O$. One section was examined from each block.

The concentration of neutrophils relative to $R B C$ in the capillaries ( $<20 \mu \mathrm{m}$ in diameter) and the small vessels (20 
I. Calculations using physiologic and radiolabeled neutrophil data:

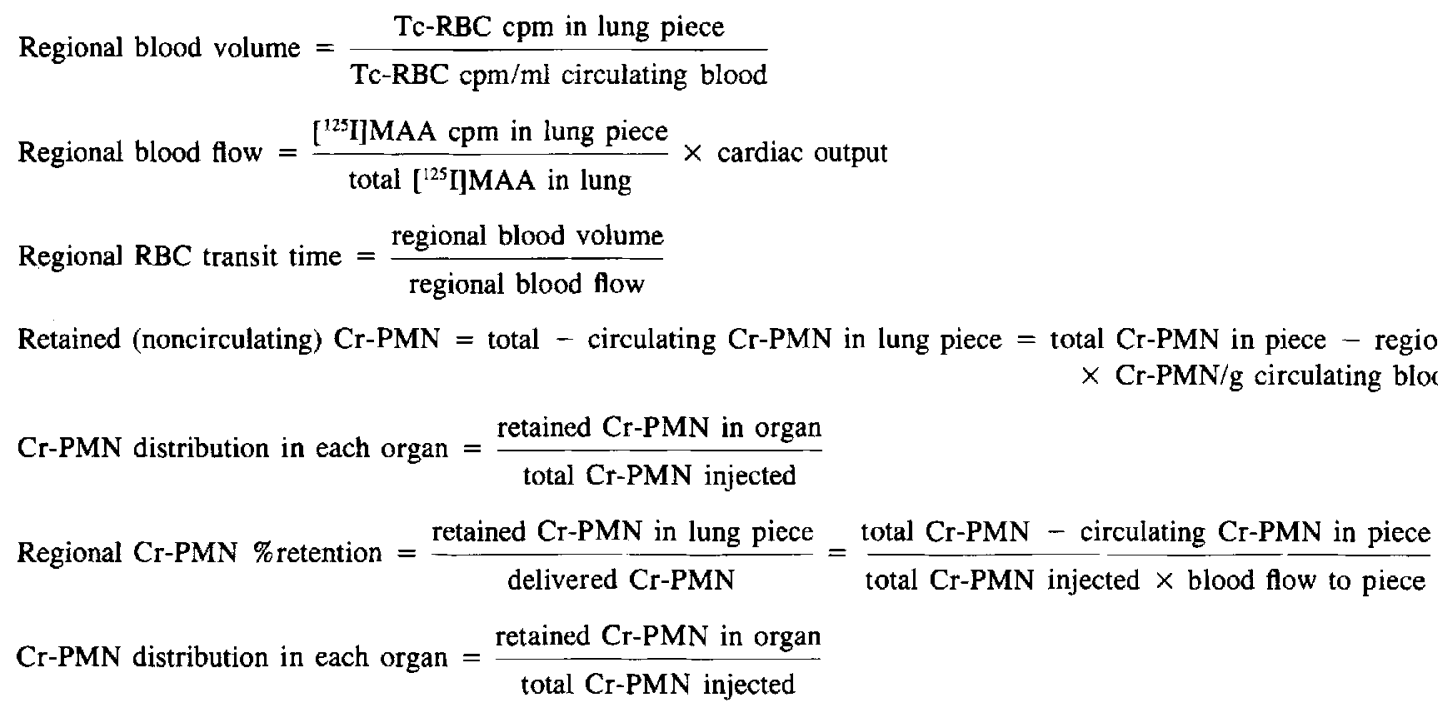

II. Morphometric quantitation of leukocyte numbers in lung:

Increase in leukocyte concentration in microvasculature of lung $=\mathrm{R}=\frac{\text { leukocytes }}{\text { lung }} / \mathrm{RBC}_{\text {luag }}$
Nukocytes blood $_{\text {blo }} / \mathrm{RBC}_{\mathrm{blod}}$
Number of leukocytes in microvasculature $=\mathrm{R} \times \frac{\text { leukocytes }}{\mathrm{ml} \text { circulating blood }} \times$ blood volume of capillaries or small vessels

to $50 \mu \mathrm{m}$ in diameter) was quantitated by counting the number of neutrophils and RBC in 10 randomly selected fields of alveolar capillaries and 10 small vessels from each block $(6,9)$. Sixty fields of alveolar vessels and 60 small vessels were examined from each rabbit ( $n=7$ in each group). The ratio of neutrophil:RBC was calculated for each vessel size. The fold increase in the concentration of neutrophils in the microvasculature of the lung relative to the circulating blood (Table 1) was calculated by dividing the neutrophil:RBC ratio in the capillaries or small vessels by this ratio in the systemic blood obtained immediately before the animal was killed. The total number of neutrophils (Table 1) was calculated by multiplying this fold increase in neutrophils times the neutrophil count $/ \mathrm{ml}$ systemic blood times the total blood volume of the lung times the fraction of the total blood volume present in the capillaries or the small vessels (capillary blood volume, $58 \%$; small vessel volume, $8 \%$ [7, 20, Table 1]). The size of the circulating pool of neutrophils was determined by multiplying the number of neutrophils/ml blood times the circulating blood volume $(60 \mathrm{ml} / \mathrm{kg}$ body weight) and was compared with the size of the neutrophil pool in the lung. The mononuclear cells (lymphocytes and monocytes) were quantitated in a similar manner.

\section{Statistics}

The Cr-PMN percent retention was compared with the $\mathrm{RBC}$ transit time by determining the linear regression that best fit the data from the 24 lung pieces for each animal. The maximum likelihood mean regression line and the $95 \%$ confidence intervals were then estimated for each group from the regression for each animal (21). The calculations were performed using Gauss version 2, revision 18 (Aptech Systems, Kent, WA).

The cardiac outputs, pulmonary blood volumes, mean RBC transit times, mean Cr-PMN retentions, organ recoveries of Cr-PMN, and the numbers of leukocytes in each vascular compartment of the lung were compared between the groups using a one-way ANOVA. When the data were not normally distributed, a log transformation was used. After finding overall differences, multiple contrasts were used to

TABLE 2

Effect of $m A b 60.3$ on circulating leukocyte counts*

\begin{tabular}{llc}
\hline & Before Pretreatment & 30 min after Pretreatment \\
\hline Group 1 & $7.4 \pm 1.3 \times 10^{6} / \mathrm{ml}$ & $6.6 \pm 0.8 \times 10^{6} / \mathrm{ml}$ \\
Group 2 & $6.6 \pm 0.9 \times 10^{6} / \mathrm{ml}$ & $5.2 \pm 0.6 \times 10^{6} / \mathrm{ml}$ \\
Group 3 & $6.4 \pm 0.6 \times 10^{6} / \mathrm{ml}$ & $5.5 \pm 0.5 \times 10^{6} / \mathrm{ml}$
\end{tabular}

* The circulating WBC counts before and after treatment with saline (groups 1 and 2) or mAb 60.3 (group 3) were not significantly different. Group 1: animals pretreated with saline that received infusions of saline. Group 2: animals pretreated with saline that received infusions of ZAP. Group 3: animals pretreated with $\mathrm{mAb} 60.3$ that received infusions of ZAP. 
determine which groups were significantly different from each other. The sequentially rejective Bonferroni test was applied to assess the significance of these contrasts (22). The frequency distributions of regional RBC transit times in each piece of lung were compared between groups by constructing cumulative frequency distributions and applying the Kolmogorov-Smirnoff test (23). The leukocyte, neutrophil, mononuclear cell, and platelet counts at each time point were compared between groups and over time using a generalized linear model for repeated measurements (24). A probability greater than 0.05 was considered to be significant. The data are expressed as mean \pm SEM.

\section{Results}

Injection of mAb 60.3 had no significant effect on the leukocyte count when compared with injection of saline (Table 2). The total leukocyte count fell within 1 min of ZAP infusion in both the saline-pretreated (group 2) and the mAb 60.3pretreated rabbits (group 3 ) and remained constant in the animals that received saline infusions (group 1). This decrease was due primarily to a fall in circulating neutrophils that occurred to a similar degree over the same time period in both groups (Figure 1, upper panel). The decrease was prolonged in the saline-pretreated animals and persisted throughout the entire $15 \mathrm{~min}$. In contrast, the neutropenia was transient in the $\mathrm{mAb}$ 60.3-pretreated animals and began to increase by $7 \mathrm{~min}$ despite continuous infusion of ZAP (Figure 1, upper panel).

The mononuclear cell count was decreased by infusion of ZAP (group 2; Figure 1, lower panel) but to a lesser degree than the neutrophil counts. Pretreatment with mAb 60.3 both slowed and partially inhibited this fall (Figure 1, lower panel).

The recovery of Cr-PMN that were circulating in the blood at 2 and 10 min after injection was reduced in the rabbits that received ZAP infusion (group 2) compared with saline (group 1; Table 3). The 2-min recovery of $\mathrm{Cr}-\mathrm{PMN}$ in the animals that were pretreated with mAb 60.3 (group 3) was similar to the saline-pretreated group after ZAP infusion (group 2), but the 10-min recovery after $\mathrm{mAb} 60.3$ pretreatment was similar to the control animals that received saline infusions (group 1).

The recovery of noncirculating Cr-PMN within the lung was $44 \pm 3 \%$ in control animals that received saline infusions and increased to $72 \pm 2 \%$ after ZAP infusion (Table
Figure 1. Changes in the circulating leukocyte and platelet counts during infusion of ZAP or saline. Group 1 (solid circles) was pretreated with saline and received infusions of saline. Group 2 (open circles) was pretreated with saline and received infusions of ZAP. Group 3 (open squares) was pretreated with mAb 60.3 and received infusions of ZAP. The data are shown as mean \pm SEM. Upper panel: Neutrophil counts. Both groups 2 and 3 showed a significant decrease in neutrophil counts when compared with group 1 at all time points between 1 and $15 \mathrm{~min}$. The fall in group 3 was similar to the fall in group 2 at 1,2 , and $4 \mathrm{~min}$ but was less at $7 \mathrm{~min}(P<0.001)$ and continued to return toward baseline values at 15 $\min (P<0.001)$. Lower panel: Mononuclear cell counts. There was a significant decrease in mononuclear cell counts in group 2 compared with group 1 at all time points between 1 and $15 \mathrm{~min}$ $(P<0.001)$. Group 3 showed a significant fall only at 2,4 , and $7 \mathrm{~min}$ when compared with group 1 $(P<0.001)$. When compared with group 2, the fall was significantly less at all time points between 1 and $15 \mathrm{~min}$.
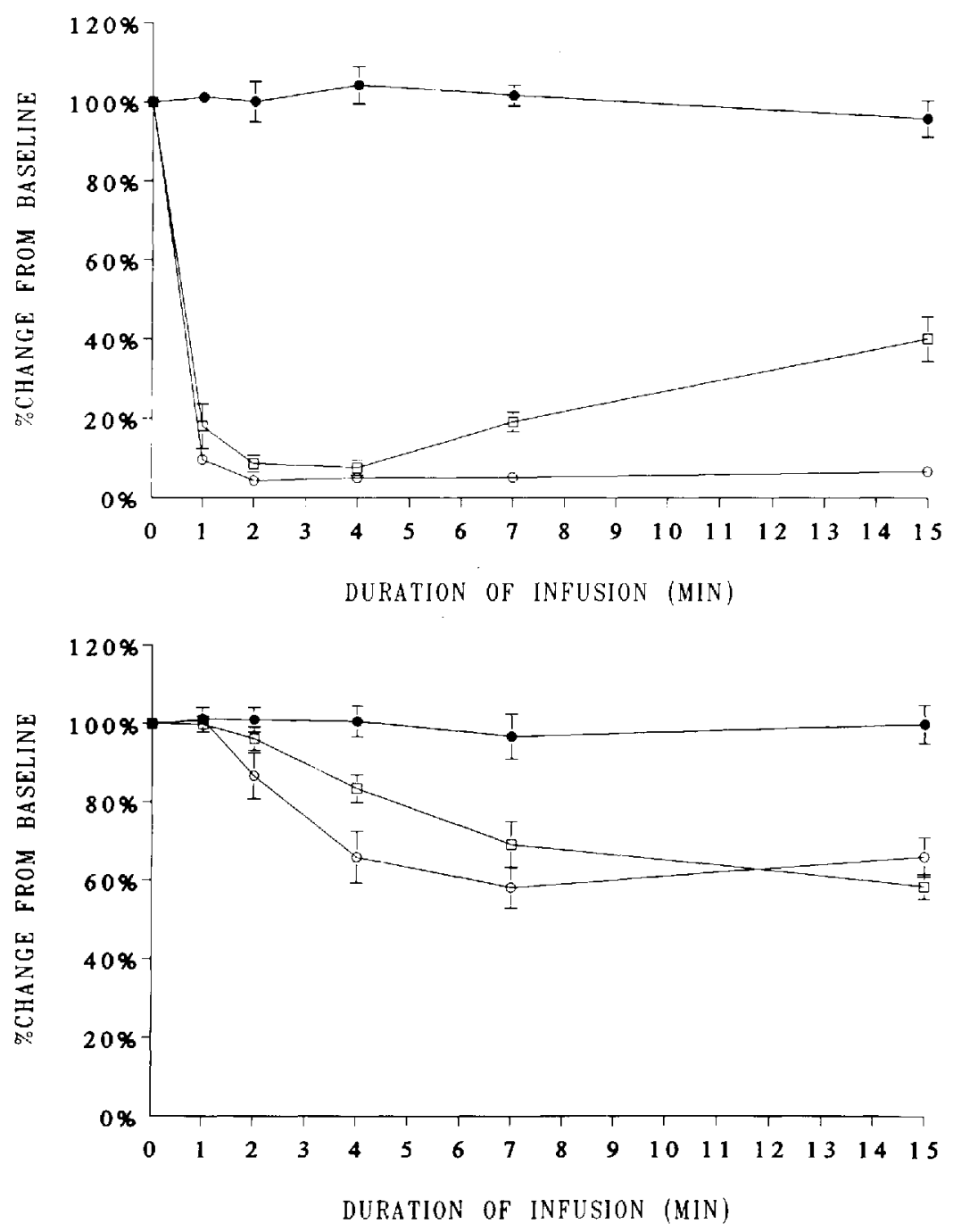
TABLE 3

\begin{tabular}{|c|c|c|c|}
\hline & Group 1 & Group 2 & Group 3 \\
\hline \multicolumn{4}{|l|}{ After $2 \mathrm{~min}$ of circulation } \\
\hline Blood & $4.8 \pm 0.5$ & $1.6 \pm 0.4^{\dagger}$ & $3.4 \pm 0.4$ \\
\hline \multicolumn{4}{|c|}{ After 10 min of circulation } \\
\hline Lung & $44.3 \pm 4.0$ & $72.0 \pm 1.8^{\ddagger}$ & $40.5 \pm 2.5$ \\
\hline Blood & $5.3 \pm 0.5$ & $1.4 \pm 0.1 末$ & $4.3 \pm 0.1$ \\
\hline Liver & $24.4 \pm 2.7$ & $4.2 \pm 0.7 \ddagger$ & $27.5 \pm 3.0$ \\
\hline Spleen & $1.0 \pm 0.3$ & $0.1 \pm 0.03 \mp$ & $1.1 \pm 0.3$ \\
\hline Kidney & $1.4 \pm 0.2$ & $2.1 \pm 0.6$ & $2.3 \pm 0.2$ \\
\hline Total & $76.4 \pm 2.0$ & $79.8 \pm 1.2$ & $75.7 \pm 1.9$ \\
\hline Plasmas & $3.7 \pm 0.8$ & $3.5 \pm 0.4$ & $2.6 \pm 0.3$ \\
\hline Marg:Circ pool sizell & $9.2 \pm 1.7$ & $55.0 \pm 4.4 \neq$ & $9.5 \pm 0.7$ \\
\hline
\end{tabular}

Definition of abbreviations: Marg = marginated; Circ = circulating.

* The percentage of the injected $\mathrm{Cr}$-PMN recovered in each organ. Group 1: animals pretreated with saline that received infusions of saline. Group 2: animals pretreated with saline that received infusions of ZAP. Group 3: animals pretreated with $\mathrm{MAb} 60.3$ that received infusions of ZAP. Mean $\pm \operatorname{SEM}(n=7$ in each group).

$\dagger$ Significantly different from group $1(P<0.05)$ but not group 3.

₹ Significantly different from group 1 and group $3(P<0.05)$.

$\$$ The percentage of ${ }^{51}$ chromium counts that were free in the plasma.

II The ratio of the marginated to circulating $\mathrm{Cr}_{-}$PMN pool size.

3). In the mAb 60.3-pretreated animals that received ZAP infusions, the recovery of noncirculating Cr-PMN was $40 \pm$ $3 \%$, which was similar to that of control animals. The organ distribution in the blood, liver, and spleen was also similar to control animals, whereas the ZAP-treated animals that did not receive $\mathrm{mAb} 60.3$ showed lower recoveries in these organs. The total recovery was similar in all three groups (Table 3).

The pulmonary blood volumes, cardiac outputs, and RBC transit times were similar in all three groups (Table 4). The frequency distribution of RBC transit time within regions of the lung also was not different between the groups.

The relationship between RBC transit time and Cr-PMN percent retention for each piece of lung tissue is shown in
TABLE 4

RBC transit time and Cr-PMN \% retention*

\begin{tabular}{lccc}
\hline & Group I & Group 2 & Group 3 \\
\hline $\begin{array}{l}\text { Pulmonary blood } \\
\text { volume (ml) }\end{array}$ & $5.53 \pm 0.55$ & $6.07 \pm 0.49$ & $5.72 \pm 0.44$ \\
$\begin{array}{c}\text { Cardiac output } \\
(\mathrm{ml} / \mathrm{min})\end{array}$ \\
$\begin{array}{c}\text { RBC transit } \\
\text { time (s) }\end{array}$ & $308 \pm 24$ & $336 \pm 23$ & $325 \pm 30$ \\
$\begin{array}{c}\text { Cr-PMN \% } \\
\text { retention }\end{array}$ & $1.07 \pm 0.07$ & $1.10 \pm 0.07$ & $1.08 \pm 0.07$ \\
\hline
\end{tabular}

* Group 1: animals pretreated with saline that received infusions of saline. Group 2: animals pretreated with saline that received infusions of ZAP. Group 3: animals pretreated with mAb 60.3 that received infusions of ZAP. Mean \pm $\operatorname{SEM}(n=7$ in each group).

† Significantly different from group 1 and group $3(P<0.001)$.

Figure 2. In all three groups, the Cr-PMN percent retention increased as the RBC transit time lengthened, and the weighted mean regression line showed a similar slope (Table 4). However, the Cr-PMN percent retention was higher in group 2, which received saline pretreatment and ZAP infusions than in groups 1 or 3 when regions of the lung that had the same RBC transit time were compared. Pretreatment with mAb 60.3 completely inhibited the ZAP-induced increase in $\mathrm{Cr}-\mathrm{PMN}$ retention at all RBC transit times. This difference between group 2 and groups 1 and 3 is reflected in the $y$-intercept of the weighted mean regression lines and in the mean Cr-PMN percent retention (Table 4).

Histologic quantification of the total numbers of neutrophils and mononuclear leukocytes in the pulmonary vasculature confirmed that there was a large marginated pool of both cell types in normal lungs (Tables 5 and 6). The ratio of neutrophils:RBC showed that a 15-min infusion of ZAP induced a large increase in neutrophils within both the alveolar capillaries (4.9-fold) and the small vessels (7.0-fold) (Table 5). Pretreatment with mAb 60.3 inhibited this increase by $80 \%$ in the capillaries and by $95 \%$ in the small vessels. In addi-

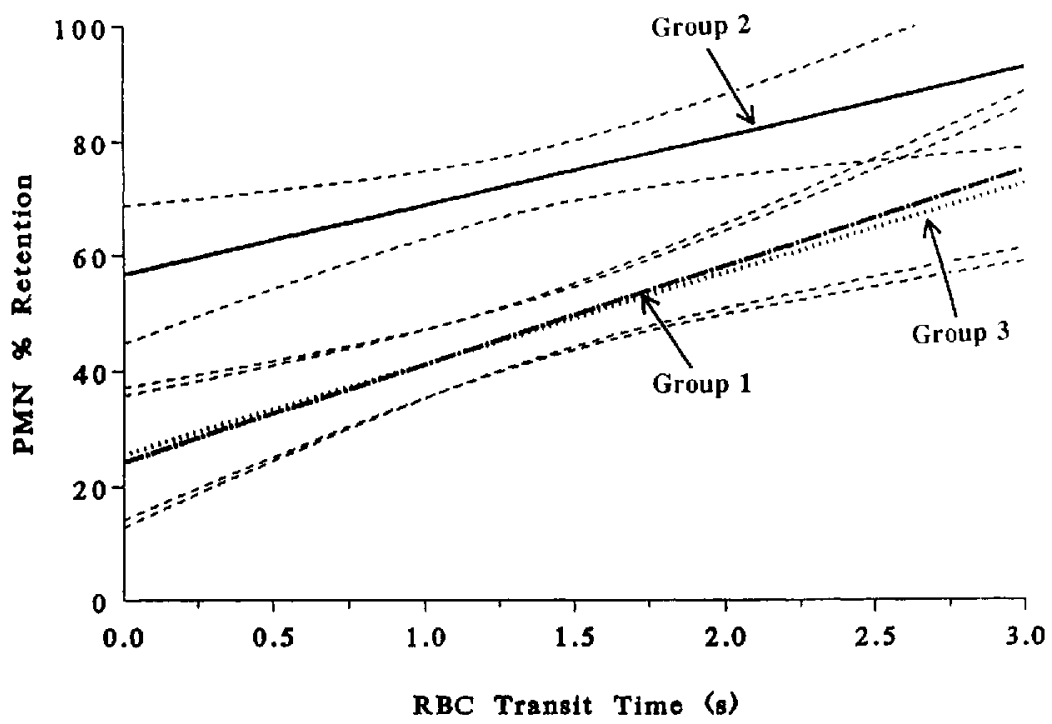

Figure 2. The relationship between Cr-PMN percent retention and RBC transit time. The regression lines obtained using maximum likelihood techniques and the $95 \%$ confidence intervals are shown for each group. In all three groups, there was an increase in Cr-PMN percent retention as RBC transit time increased. Infusion of ZAP (group 2, solid line) increased the Cr-PMN percent retention at all RBC transit times compared with the controls (group 1, short-long dashed line) $(P<0.001)$. These ZAP-induced changes in retention were completely inhibited by pretreatment of the animals with mAb 60.3 (group 3, dotted line). The equations describing the regression lines are: group $1, y=15.6 x+25.7$; group $2, y=12.1 x+$ 56.7; group $3, y=16.8 x+24.4$. 
TABLE 5

Relative concentration of leukocytes to $R B C$ in the alveolar capillaries and the small vessels $(20 \text { to } 50 \mu \mathrm{m})^{*}$

\begin{tabular}{lccc}
\hline & Group 1 & Group 2 & Group 3 \\
\hline Neutrophils:RBC $\left(\times 10^{-3}\right)$ & \multicolumn{3}{c}{} \\
Capillaries & $17.8 \pm 1.0$ & $87.5 \pm 2.8^{\dagger}$ & $31.8 \pm 3.9 \neq$ \\
Small vessels & $3.4 \pm 0.7$ & $23.7 \pm 2.4^{\dagger}$ & $4.5 \pm 1.0^{\ddagger}$ \\
Fold increase compared with group $1 \mathrm{in:}$ & \\
$\quad$ Capillaries & 4.9 & 1.8 \\
$\quad$ Small vessels & 7.0 & 1.3 \\
Mononuclear cells:RBC $\left(\times 10^{-3}\right)$ & & \\
Capillaries & $10.0 \pm 0.8$ & $25.4 \pm 1.1^{\dagger}$ & $15.4 \pm 1.2 \ddagger$ \\
Small vessels & $0.8 \pm 0.5$ & $1.4 \pm 0.7$ & $0.4 \pm 0.4$ \\
Fold increase compared with group 1 in: & \\
$\quad$ Capillaries & & 2.5 & 1.5 \\
$\quad$ Small vessels & & 1.7 & 0.5 \\
\hline
\end{tabular}

* Group 1: animals pretreated with saline that received infusions of saline. Group 2: animals pretreated with saline that received infusions of ZAP. Group 3: animals pretreated with $\mathrm{mAb} 60.3$ that received infusions of ZAP.

+ Significantly different from group 1 and group $3(P<0.01)$.

₹ Significantly different from group 1 and group $2(P<0.01)$. tion, ZAP infusion also increased the numbers of mononuclear cells within the alveolar capillaries, and $\mathrm{mAb} 60.3$ also inhibited this increase by about $70 \%$ (Table 5). ZAP infusion did not cause a significant increase in mononuclear cells within the small vessels.

The changes induced by ZAP in the size of the total marginated pool and in the concentration of leukocytes $/ \mathrm{ml}$ blood are shown in Table 6. In the control animals, the normal marginated pool contained 1.4 times the number of neutrophils that were in the entire circulating pool. This margination was due primarily to a 36 -fold increase in concentration of neutrophils within the capillary bed when compared with arterial blood and a smaller increase of 6.4-fold in the small vessels (Table 6). However, because of the large portion of blood that is in the capillaries, these vessels contained $97 \%$ of the neutrophils. Infusion of ZAP caused a 4.4 -fold increase in the number of neutrophils within capillaries and a 6.4-fold increase in the number within small vessels. Pretreatment with mAb 60.3 completely inhibited the increase in neutrophil concentration within the small vessels and prevented the increase in the capillary bed by $83 \%$ (Table 6 ).

TABLE 6

Number of leukocytes in the pulmonary vasculature*

\begin{tabular}{|c|c|c|c|}
\hline & Group 1 & Group 2 & Group 3 \\
\hline \multicolumn{4}{|l|}{ Neutrophils $\left(\times 10^{6}\right)$} \\
\hline \multicolumn{4}{|l|}{ Circulating blood } \\
\hline Number/ml blood & $2.8 \pm 0.5$ & $0.19 \pm 0.04^{\dagger}$ & $0.81 \pm 0.12 \ddagger$ \\
\hline Total pool size & $264 \pm 47$ & $15.3 \pm 4.4^{\dagger}$ & $65.9 \pm 11.6$ \\
\hline \multicolumn{4}{|l|}{ Pulmonary capillaries } \\
\hline Total number & $292 \pm 23$ & $1,430 \pm 94^{\dagger}$ & $469 \pm 50 \ddagger$ \\
\hline Number/ml cap. blood & $90 \pm 6$ & $397 \pm 13^{\dagger}$ & $141 \pm 17 \ddagger$ \\
\hline Fold increase in cap. 8 & $36 \pm 5$ & $2,671 \pm 451 \dagger$ & $205 \pm 37 \ddagger$ \\
\hline \multicolumn{4}{|l|}{ Pulmonary small vessels } \\
\hline Total number & $7.5 \pm 1.7$ & $52.4 \pm 6.6^{\dagger}$ & $9.3 \pm 2.3$ \\
\hline Number/ml sm. ves. blood & $16.6 \pm 3.6$ & $107 \pm 10^{\dagger}$ & $19.7 \pm 4.2$ \\
\hline Fold increase in sm. ves. & $6.4 \pm 1.4$ & $697 \pm 111^{\dagger}$ & $29.1 \pm 7.0$ \\
\hline Marg:Circ pool sizell & $1.4 \pm 0.3$ & $132 \pm 24^{\dagger}$ & $9.4 \pm 2.1 \pm$ \\
\hline$\%$ in capillaries & $97 \pm 1 \%$ & $96 \pm 0.4 \%$ & $98 \pm 0.3 \%$ \\
\hline$\%$ in small vessels & $3 \pm 0.5 \%$ & $4 \pm 0.4 \%$ & $2 \pm 0.3 \%$ \\
\hline \multicolumn{4}{|l|}{ Mononuclear cells $\left(\times 10^{6}\right)$} \\
\hline \multicolumn{4}{|l|}{ Circulating blood } \\
\hline Number/ml blood & $3.9 \pm 0.5$ & $1.8 \pm 0.2^{\dagger}$ & $3.0 \pm 0.3$ \\
\hline Total pool size & $362 \pm 50$ & $144 \pm 21^{\dagger}$ & $240 \pm 29$ \\
\hline \multicolumn{4}{|l|}{ Pulmonary capillaries } \\
\hline Total number & $165 \pm 18$ & $419 \pm 37^{\dagger}$ & $229 \pm 23 \ddagger$ \\
\hline Number/ml cap. blood & $50 \pm 3$ & $115 \pm 5^{\dagger}$ & $68 \pm 6 \ddagger$ \\
\hline Fold increase in cap. 8 & $14 \pm 2$ & $69 \pm 9^{\dagger}$ & $25 \pm 4 \ddagger$ \\
\hline \multicolumn{4}{|l|}{ Pulmonary small vessels } \\
\hline Total number & $1.5 \pm 1.0$ & $3.1 \pm 1.5$ & $0.6 \pm 0.6$ \\
\hline Number $/ \mathrm{ml}$ sm. ves. blood & $4.1 \pm 2.7$ & $6.1 \pm 2.9$ & $1.8 \pm 1.8$ \\
\hline Fold increase in sm. ves. $\$$ & $1.3 \pm 0.8$ & $3.2 \pm 1.6$ & $0.6 \pm 0.6$ \\
\hline Marg:Circ pool sizell & $0.52 \pm 0.12$ & $3.4 \pm 0.4$ & $1.6 \pm 0.2$ \\
\hline
\end{tabular}

Definition of abbreviations: cap. $=$ capillaries; sm. ves. $=$ small vessels $(20$ to $50 \mu \mathrm{m})$; Marg $=$ marginated; Circ $=$ circulating.

* Group 1: animals pretreated with saline that received infusions of saline. Group 2: animals pretreated with saline that received infusions of ZAP. Group 3: animals pretreated with mAb 60.3 that received infusions of ZAP.

$\dagger$ Significantly different from group 1 and group $3(P<0.05)$.

\# Significantly different from group 1 and group $2(P<0.05)$.

$\$$ Fold increase in concentration $/ \mathrm{ml}$ blood when compared to the concentration of leukocytes in the circulating blood of the same animal.

I| Ratio of the size of the marginating (capillary plus small vessel) pool to the circulating pool. 
The pulmonary vasculature also contained a marginated pool of mononuclear cells that was 0.52 times the size of the entire circulating pool. The mononuclear cells showed an increase in concentration within capillaries that measured 14 times that of arterial blood (Table 6). Infusion of ZAP increased this marginated pool 2.3 times, and the accumulation was inhibited by pretreatment with mAb 60.3 (Table 6).

\section{Discussion}

This study and others $(9,25-27)$ have shown that infusion of activated plasma induced profound neutropenia and a moderate decrease in mononuclear cells within $1 \mathrm{~min}$ that persisted for the 15-min duration of the infusion. This fall in circulating cells was due to the accumulation of neutrophils and mononuclear cells within both the capillaries and the small vessels of the lung, demonstrated both by histologic counts of native leukocytes and by radiolabeled neutrophils. Pretreatment with an anti-CD18 antibody did not alter either the rate of onset or the degree of the rapid fall in circulating neutrophils. However, mAb 60.3 did inhibit the persistent neutropenia that occurred between 7 and $15 \mathrm{~min}$ of ZAP infusion. In addition, this pretreatment almost completely inhibited the continued sequestration and resultant accumulation of neutrophils within the pulmonary microvasculature after $15 \mathrm{~min}$. These data suggest that the changes in neutrophil kinetics induced by infusion of activated plasma occurred through two separate mechanisms. The first process produced the initial neutropenia and intravascular sequestration within $1 \mathrm{~min}$ by mechanisms that do not involve CD11/CD18-mediated adhesion. The second process resulted in prolonged neutropenia and massive neutrophil accumulation in the lung by $15 \mathrm{~min}$ and required CD11/ CD18-mediated adhesion. The histologic studies show that CD11/CD18-mediated adhesion was completely responsible for the increase in neutrophil concentration within the vessels that measure 20 to $50 \mu \mathrm{m}$ in diameter and was a major factor in increasing the concentration within capillaries. These data are supported by work of Yoder and associates (28), who found that neutrophils from a dog with a genetic deficiency of CD11/CD18 did not sequester in the lungs of a normal dog after activation by ZAP. Our data are also supported by the studies of Lundberg and Wright (29), who found that transient formylmethionylleucylphenylalanineinduced neutropenia was not inhibited by pretreatment using an anti-CD18 antibody.

The rapid sequestration that was CD11/CD18-independent might be due to an alternative adhesion system or to a decrease in the deformative ability of the neutrophil. Several CD18-independent adhesion systems have been described. The receptor for the first system is L-selectin (LECAM-1, LAM-1, Mel-14 antigen) (30-37) on the neutrophil that interacts with endothelial ligands that likely include E-selectin (ELAM-1) and P-selectin (GMP-140, CD62) (36, 37). In contrast to CD11/CD18, which is expressed in low quantities on the plasma membrane of unactivated neutrophils and is upregulated during activation, $\mathrm{L}$-selectin is expressed on the surface of the neutrophil when the cell is circulating and is downregulated when the neutrophil responds to an inflammatory stimulus (30-32). L-selectin and CD11/CD18 may interact sequentially or collaboratively to mediate adhesion and migration (34-37). Whether or not L-selectin plays any role in the initial fall in circulating neutrophils seen in these experiments remains to be determined.

Another CDI 1/CD18-independent system has been shown to mediate neutrophil emigration into the alveolar spaces of the lung in response to Streptococcus pneumoniae and hydrochloric acid (13). Although these stimuli elicit CD11/ CD18-dependent neutrophil emigration in the systemic circulation, a separate, as yet undefined adhesion system is responsible for neutrophil emigration in the pulmonary circulation. It is possible that this system could play a role in ZAP-induced sequestration. However, until a specific antibody that inhibits the function of this system becomes available, this question cannot be answered.

Although CD11/CD18-independent adhesion may be important in the initial neutropenia observed in these experiments, it is likely that a decrease in the deformability of the neutrophil is at least partially responsible for this neutropenia. The unactivated neutrophil is approximately 1,000 times less deformable than the RBC (1-5). The normal marginated pool of neutrophils that is present within the lung capillary bed forms because the poorly deformable neutrophils, which measure $6.4 \mu \mathrm{m}$ in diameter in rabbits, must negotiate capillary pathways that consist of many narrow capillary segments averaging $5.8 \pm 0.4 \mu \mathrm{m}$ in diameter (38). Previous data from our laboratory have shown that the multisegmented interconnecting structure of this bed allows the RBC to bypass the segments temporarily obstructed by a neutrophil, concentrating the neutrophils relative to the $\operatorname{RBC}(5-7,9)$. Direct measurements through a pleural window obtained by Lien and co-workers in dogs showed that the median neutrophil transit time was $26.1 \mathrm{~s}$ (mean transit time, $6.1 \mathrm{~min}$ ) whereas the plasma transit time was $1.4 \pm 0.3 \mathrm{~s}(8)$. If activated neutrophils became even less deformable and required more time to pass through narrow capillaries, the circulating neutrophil count would fall and the size of the lung marginated pool would rise until a new equilibrium between the marginating and circulating pools was established. In vitro studies support this hypothesis by showing that many stimuli that activated neutrophils produced a rapid decrease in deformability $(10,39-41)$, which was associated with an increase in the amount of f-actin present within the cytoskeleton (10) and was not prevented by pretreatment with the anti-CD18 antibody, mAb 60.3 (10).

This study indicates that the mechanism by which the initial neutrophil sequestration occurred was sufficient to maintain the neutropenia and tissue sequestration for only a brief period of time. Several lines of evidence support this conclusion. First, the circulating neutrophil counts in the mAb 60.3-pretreated animals were increasing toward baseline levels despite continuous infusion of ZAP. Second, although the recovery of Cr-PMN in the blood at 2 min was low, similar to that in the saline-pretreated animals during ZAP infusion (group 2), by $10 \mathrm{~min}$ the recovery had increased to values similar to control animals (group 1). Finally, the ZAPinduced increase in both the Cr-PMN percent retention and the accumulation of native neutrophils within the pulmonary vasculature was inhibited after 10 to $15 \mathrm{~min}$ of ZAP infusion. These data indicate that the CD18-independent sequestration of neutrophils is short-lived, lasting between 4 and $7 \mathrm{~min}$ when CD18 is blocked. These changes in the neutrophil 
count could be due to the establishment of a new equilibrium between the marginating and circulating neutrophil pools after a ZAP-induced increase in neutrophil transit time. Alternatively, the changes in neutrophil deformability or adhesion might be reversible.

If 4 to $7 \mathrm{~min}$ represented the time required for a new equilibrium to occur between marginating and circulating pools of neutrophils that had become less deformable, then the size of the marginated pool in the lung would be increased. In fact, our data quantitating the size of the marginated pool of native neutrophils in the capillaries indicate that although pretreatment with mAb 60.3 markedly inhibited the ZAPinduced increase in margination, this pool size was increased by $57 \%$ in these rabbits compared with control animals (group 1,90 \pm 6 neutrophils/ml capillary blood versus group 3, $141 \pm 17$ neutrophils/ml capillary blood; Table 6). In addition, mAb 60.3 completely prevented the increased neutrophil concentration in the small vessels where adhesion rather than decreased deformability is far more likely to mediate neutrophil accumulation. Taken together, these data suggest that the mechanism of the initial sequestration was due to a change in the deformability of the neutrophil and that the equilibrium between the marginated and circulating pools was shifted toward an increase in the size of the marginated pool. However, a change in the CD18-independent adhesive characteristics of the neutrophil by ZAP that is reversible cannot be completely excluded.

In normal lungs, the marginated pool of neutrophils was $1.4 \pm 0.3$ times the size of the circulating pool (Table 6). The marginating and circulating pools are in equilibrium because the neutrophil counts in arterial and venous blood samples are the same (42). This means that the number of neutrophils that enter the marginated pool is equal to the number that are released. When activated plasma is infused, there is a marked shift of neutrophils from the circulating pool into the marginated pool leading to accumulation of cells within the lung microvasculature $(9,28)$. In fact, our data (Table 6) show that the number of neutrophils that accumulate within the lungs during the infusion is far greater than the number that were present in the circulation before the infusion $(1,430$ $\times 10^{6}$ in the capillaries after ZAP $+52.4 \times 10^{6}$ in the small vessels $=1,482 \times 10^{6}$ total after ZAP compared with $264 \times 10^{6}$ circulating neutrophils $+292 \times 10^{6}$ in the capillaries $+7.5 \times 10^{6}$ in the small vessels $=563 \times 10^{6}$ in the circulating and marginated pools of normal rabbits; Table 6). This suggests that ZAP infusion was associated with the release of a second marginated pool, such as the bone marrow pool (43), which is sequestered in the lung almost immediately upon entering the circulation. The stimulus for the release of neutrophils could be the low circulating counts themselves or a stimulus in the ZAP, possibly the C3e fragment of complement protein 3 (44). Because the release occurred very rapidly during the course of this experiment, cytokines that require protein synthesis are not reasonable candidates.

In contrast to neutrophils, the initial fall in mononuclear cells induced by infusion of ZAP was not as great (Figure 1, upper panel, compared with Figure 1, lower panel). In addition, inhibition of CD18-dependent adhesion both delayed and partially prevented the fall in mononuclear cells. The capillary accumulation of these cells was also inhibited but to a slightly lesser degree than the neutrophils (Table 6). These data suggest that the sequestration and accumulation of mononuclear cells occurred through several mechanisms and may reflect the variety of cell populations within this group.

In conclusion, these data show that neutrophil accumulation within the lung induced by infusion of ZAP is a two-step process. The first process occurs through a CD18-independent system, produces profound neutropenia by $1 \mathrm{~min}$, and can sequester neutrophils for 4 to $7 \mathrm{~min}$. A second CD18-dependent mechanism accounts for the prolonged sequestration of neutrophils that increases the marginated pool 5-fold after 15 min of ZAP infusion. We suggest that the CD18-independent step may be required to slow the neutrophils in preparation for CD18-mediated adhesion to the endothelium and may occur through either a stimulus-induced decrease in neutrophil deformability or a CD18-independent adhesion system.

Acknowledgments: The writer wishes to thank Dr. James C. Hogg for invaluable discussions and for thoughtful criticism of the manuscript. The monoclonal antibody 60.3 was kindly provided by Dr. John M. Harlan, University of Washington, who reviewed the manuscript. I am also grateful to Dr. Wiltz W. Wagner, Jr., Indiana University, for reviewing the manuscript. Dean English provided excellent technical assistance, Barry $\mathbf{R}$. Wiggs performed the statistical analysis, and Harvey $O$. Coxson prepared the histologic sections. This work was supported by the British Columbia Health Care Research Foundation, the $B C$ and Yukon Heart Foundation, Council of Canada 4219, and USPHS HL 30542. Dr. Doerschuk is the recipient of a Parker B. Francis Fellowship in Pulmonary Research and an American Lung Association Career Investigator Award.

\section{References}

1. Chien, S. 1985. Role of blood cells in microcirculatory regulation. Microvasc. Res. 29:129-151.

2. Schmid-Schonbein, G. W., S. Usami, R. Skalak, and S. Chien. 1980. The interaction of leukocytes and erythrocytes in capillary and postcapillary vessels. Microvasc. Res. 19:45-70.

3. Chien, S., G. Schmid-Schonbein, K.-L. P. Sung, E. A. Schmalzer, and R. Skalak. 1984. Viscoelastic properties of leukocytes. In White Cell Mechanics: Basic Science and Clinical Aspects. H. J. Meiselman, M. A. Lightman, and P. L. LaCelle, editors. Alan R. Liss, New York. 19-51.

4. Chien, S., K.-L. P. Sung, G. W. Schmid-Schonbein, R. Skalak, E. A Schmalzer, and S. Usami. 1987. Rheology of leukocytes. Ann. NY Acad. Sci. 516:333-347.

5. Hogg, J. C. 1987. Neutrophil kinetics and lung injury. Physiol. Rev. 67:1249-1295.

6. Doerschuk, C. M., M. F. Allard, B. A. Martin, A. MacKenzie, A. P. Autor, and J. C. Hogg. 1987. Marginated pool of neutrophils in rabbit lungs. J. Appl. Physiol. 63:1806-1815.

7. Hogg, J. C., T. McLean, B. A. Martin, and B. Wiggs. 1988. Erythrocyte transit and neutrophil concentration in the dog lung. J. Appl. Physiol. $65: 1217-1225$

8. Lien, D. C., W. W. Wagner, Jr., R. L. Capen et al. 1987. Physiological neutrophil sequestration in the lung: visual evidence for localization in capillaries. J. Appl. Physiol. 62:1236-1243.

9. Doerschuk, C. M., M. F. Allard, and J. C. Hogg. 1989. Neutrophil kinetics in rabbits during infusion of zymosan-activated plasma. J. Appl. Physiol. 67:88-95.

10. Worthen, G. S., B. Schwab, E. L. Elson, and G. P. Downey, 1989 Mechanics of stimulated neutrophils: cell stiffening induces retention in capillaries. Science 245:183-185.

11. Harlan, J. M., P. D. Killen, F. M. Senecal et al. 1985. The role of neutrophil membrane glycoprotein GP-150 in neutrophil adherence to endothelium in vitro. Blood $66: 167-178$.

12. Pohlman, T. H., K. A. Stanness, P. G. Beatty, H. D. Ochs, and J. M Harlan. 1986. An endothelial cell surface factor(s) induced in vitro by lipopolysaccharide, interleukin 1 , and tumor necrosis factor-alpha increases neutrophil adherence by a CDw18-dependent mechanism. $J . \mathrm{Im}$ munol. 136:4548-4553.

13. Doerschuk, C. M., R. K. Winn, H. O. Coxson, and J. M. Harlan. 1990. CD18-dependent and -independent mechanisms of neutrophil emigration in the pulmonary and systemic microcirculation of rabbits. J. Immunol. 144:2327-2333.

14. Arfors, K. E., C. Lundberg, L. Lindbom, K. Lundberg, P. G. Beatty, and J. M. Harlan. 1987. A monoclonal antibody to the membrane glycopro- 
tein complex CDw18 (LFA) inhibits PMN accumulation and plasma leakage in vivo. Blood 69:338-343.

15. Price, T. J., P. G. Beatty, and S. R. Corpuz. 1987. In vivo inhibition of neutrophil function in the rabbit using monoclonal antibody to CD18. $J$. Immunol. 139:4174-4177.

16. Nourshargh, S., M. Rampart, P. G. Hellewell et al. 1989. Accumulation of ${ }^{11}$ In-neutrophils in rabbit skin in allergic and non-allergic inflammatory reactions in vivo: inhibition by neutrophil pretreatment in vitro with a monoclonal antibody recognizing the $\mathrm{CD} 18$ antigen. J. Immunol. 142:3193-3198.

17. Beatty, P. G., J. A. Ledbetter, P. J. Martin, T. H. Price, and J. A. Hansen. 1983. Definition of a common leukocyte cell-surface antigen (Lp 95-150) associated with diverse cell-mediated immune functions. J. Immunol. 131:2913-2918.

18. Gutkowski, R. F., and H. J. Dworkin. 1974. Kit-produced ${ }^{99 \mathrm{~m}} \mathrm{Tc}$-labeled red cells for spleen imaging. J. Nucl. Med. 15:1187-1191.

19. Zierler, K. L. 1962. Circulation times and the theory of indicator dilution methods for determining blood flow and volume. In Handbook of Physiology. Circulation. Section 2, Vol. I. American Physiological Society, Washington, D.C. $585-615$

20. Doerschuk, C. M., M. L. Brumwell, H. O. Coxson, and J. C. Hogg. 1989 Neutrophil (PMN) sequestration in rabbits following infusion of complement fragments. Proceedings of the XXXI International Union of Physiological Sciences XVII:280. (Abstr. \#P3330.) Oy Liitto, Oulu, Finland.

21. Feldman, H. A. 1988. Families of lines; random effects in linear regression analysis. J. Appl. Physiol. 64:721-732.

22. Holland, B. S., and M. D. Copehaver. 1987. An improved sequentially rejective Bonferroni test procedure. Biometrics 43:417-423.

23. Conover, W. 1980. Practical Nonparametric Statistics. 2nd ed. John Wiley, Toronto. 344-384.

24. Wei, L. J., and D. O. Stram. 1988. Analysing repeated measurements with possibly missing observations by modelling marginal distribution. Stat. Med. 7:139-148.

25. O'Flaherty, J. T., P. R. Craddock, F. McCullough, A. P. Dalmasso, and H. S. Jacob. 1978. Effect of intravascular complement activation on granulocyte adhesiveness and distribution. Blood 51:731-739.

26. Henson, P. M., G. L. Larsen, R. O. Webster, B. C. Nitchel, A. H. Goins, and J. E. Henson. 1982. Pulmonary microvascular alterations and injury induced by complement fragments: synergistic effect of complement activation, neutrophil sequestration, and prostaglandins. Ann. NY Acad. Sci. $384: 287-300$.

27. Borg, T., B. Gerdin, R. Hallgren, and J. Modig. 1985. The role of polymorphonuclear leucocytes in the pulmonary dysfunction induced by complement activation. Acta Anaesthesiol. Scand. 29:231-240.

28. Yoder, M. C., L. L. Checkley, U. Giger et al. 1990. Pulmonary microcirculatory kinetics of neutrophils deficient in leukocyte adhesion-promoting glycoproteins. J. Appl. Physiol. 69:207-213.

29. Lundberg, C., and S. D. Wright. 1990 . Relation of the CD11/CD 18 family of leukocyte antigens to the transient neutropenia caused by chemoattractants. Blood 76:1240-1245.

30. Lewinsohn, D. M., R. F. Bargatze, and E. C. Butcher. 1987. Leukocyteendothelial cell recognition: evidence of a common molecular mechanism shared by neutrophils, lymphocytes, and other leukocytes. J. Immunol.
138:4313-4321.

31. Kishimoto, T. K., M. A. Jutila, E. L. Berg, and E. C. Butcher. 1989. Neutrophil Mac-1 and MEL-14 adhesion proteins inversely regulated by chemotactic factors. Science 245:1238-1241.

32. Jutila, M. A., L. Rott, E. L. Berg, and E. C. Butcher. 1989. Function and regulation of the neutrophil MEL-14 antigen in vivo: comparison with LFA-1 and MAC-1. J. Immunol. 143:3318-3324.

33. Hallmann, R., M. A. Jutila, C. W. Smith, D. C. Anderson, T. K. Kishimoto, and E. C. Butcher. 1991. The peripheral lymph node homing receptor, LECAM-1, is involved in CD18-independent adhesion of human neutrophils to endothelium. Biochem. Biophys. Res. Commun. 174: 236-243.

34. Ley, K., P. Gachtgens, C. Fennie, M. S. Singer, L. A. Lasky, and S. D. Rosen. 1991. Lectin-like cell adhesion molecule 1 mediates leukocyte rolling in mesenteric venules in vivo. Blood 77:2553-2555.

35. Von Andrian, U. H., J. D. Chambers, L. M. McEvoy, R. F. Bargatze, K.-E. Arfors, and E. C. Butcher. 1991. Two-step model of leukocyteendothelial cell interaction in inflammation: distinct roles for LECAM-1 and the leukocyte $\beta_{2}$ integrins in vivo. Proc. Natl. Acad. Sci. USA 88 7538-7542.

36. Picker, L. J., R. A. Warnock, A. R. Burns, C. M. Doerschuk, E. L. Berg, and E. C. Butcher. 1991. The neutrophil selectin LECAM-1 presents carbohydrate ligands to the vascular selectins ELAM-1 and GMP-140. Cell 66:921-933.

37. Lawrence, M. B., and T. A. Springer. 1991. Leukocytes roll on a selectin at physiologic flow rates: distinction from and prerequisite for adhesion through integrins. Cell 65:859-873.

38. Beyers, N., C. M. Doerschuk, H. O. Coxson, and J. C. Hogg. 1989. Neutrophil volume and capillary segment diameter in the rabbit, the dog and the human. Am. Rev. Respir. Dis. 139:A297. (Abstr.)

39. Nash, G. B., J. G. Jones, J. Mikita, B. Christopher, and J. A. Dormandy. 1988. Effects of preparative procedures and of cell activation on flow of white cells through micropore filters. Br. J. Haematol. 70:171-176.

40. Higson, F. H., Y. Kikuchi, O. T. G. Jones, and G. M. Hughes. 1983. Effects of respiratory-burst stimuli on the flow properties of pig blood neutrophils. J. Cell Sci. 64:323-330.

41. Cheung, A. T. W., and M. E. Miller. 1984. Chemotaxis, chemokinesis, and movement. In White Cell Mechanics: Basic Science and Clinical Aspects. H. J. Meiselman, M. A. Lightman, and P. L. LaCelle, editors. Alan R. Liss, New York. 169-193.

42. Martin, B. A., J. L. Wright, H. Thommasen, and J. C. Hogg. 1982. Effect of pulmonary blood flow on the exchange between the circulating and marginating pool of polymorphonuclear leukocytes in dog lungs. J. Clin. Invest. 69:1277-1285.

43. McKenna, P. J., D. L. Rosolia, F. Cerasoli, Jr., K. H. Albertine, and M. H Gee. 1989. Effects of intravascular complement activation on neutrophils (PMN) in blood and bone marrow in sheep. Am. Rev. Respir. Dis. 139:A297. (Abstr.)

44. Tavassoli, M. 1987. Structural alterations of marrow during inflammation. Blood Cells 13:251-261.

45. Anderson, D. C., and T. A. Springer. 1987. Leukocyte adhesion deficiency an inherited defect in the Mac-1, LFA-1, and p150,95 glycoproteins. Annu. Rev. Med. 38:175-194. 